\title{
Optimization Process for Berth and Quay-Crane Assignment in Container Terminals with Separate Piers
}

\author{
By Neven Grubisic* \\ Livia Maglic ${ }^{\dagger}$
}

The objective of this research is the study of container terminals with two separated piers within the same port basin. The main problem is how to optimize the berth and crane allocation and to minimize the overall service time for the vessels and to improve the utilization of the terminal assets. The optimization of the seaside subsystem of the container terminals combines three typical operational problems: ship-to-berth allocation, quay-crane to ship assignment and quay-crane scheduling. Due to their characteristics, they have a high correlation and should be considered together. The problem can become even more complex in the Container terminals with a different layout where quays and berths are not placed in the line or where berths are situated in different piers. In this paper, a specific methodology is presented with a focus on the optimization process. This process consists of three stages namely: initiation, allocation and adjustment. The core of the problem solutions in stage 1 is the execution of crane scheduling problem according to cargo volume and container distribution on the vessel. The result of this stage is three operational scenarios that set out two key variables: duration of the handling process and the number of cranes required. According to the results from stage 1, ship-to-berth assignment and allocation of cranes isexecuted. The practical approach implemented here, targets to high prediction, reliability and efficiency of the operational plans to satisfy the requirements of the shipping companies. This approach requires a fixed number of quay-cranes during the handling operations and high utilization rate of the cranes. The results of the overall optimization have been shown on the few examples.

Keywords: Berth allocation problem, Container terminal optimization, CT logistics, QC scheduling problem.

\section{Introduction}

On the large container terminals, harbor quays are usually placed in a straight line in order to ensure good connection with container stacking area and to ensure better berth utilization. However, port quays may be designed in different layout such as "L" or "U" shape or vessel berths may be placed on separate piers. Such is the case with port basins, where both sides of the basin are used for berthing the vessels and for handling operations (Figure 1). In such a case, we have two terminal areas with independent operations within the same container terminal. Each terminal area has its own resources, which do not correlate with those on the other side. Due to this fact, the optimization should be

\footnotetext{
* Assistant Professor, University of Rijeka, Croatia.

${ }^{\dagger}$ Senior Research Assistant, University of Rijeka, Croatia.
} 
approached in a different way, where the main goal remains the minimum time the ship in the port with equal capacity utilization of quay-cranes.

This configuration of the container terminal is more appropriate for small and medium size terminals where different size of vessels may be expected. Basin terminal type is less suitable for maneuvering large container ships, therefore ports with large annual turnover of containers, may prefer construction of straight-line quays if available.

Figure 1. Layout of Container Terminal with Separate Quays

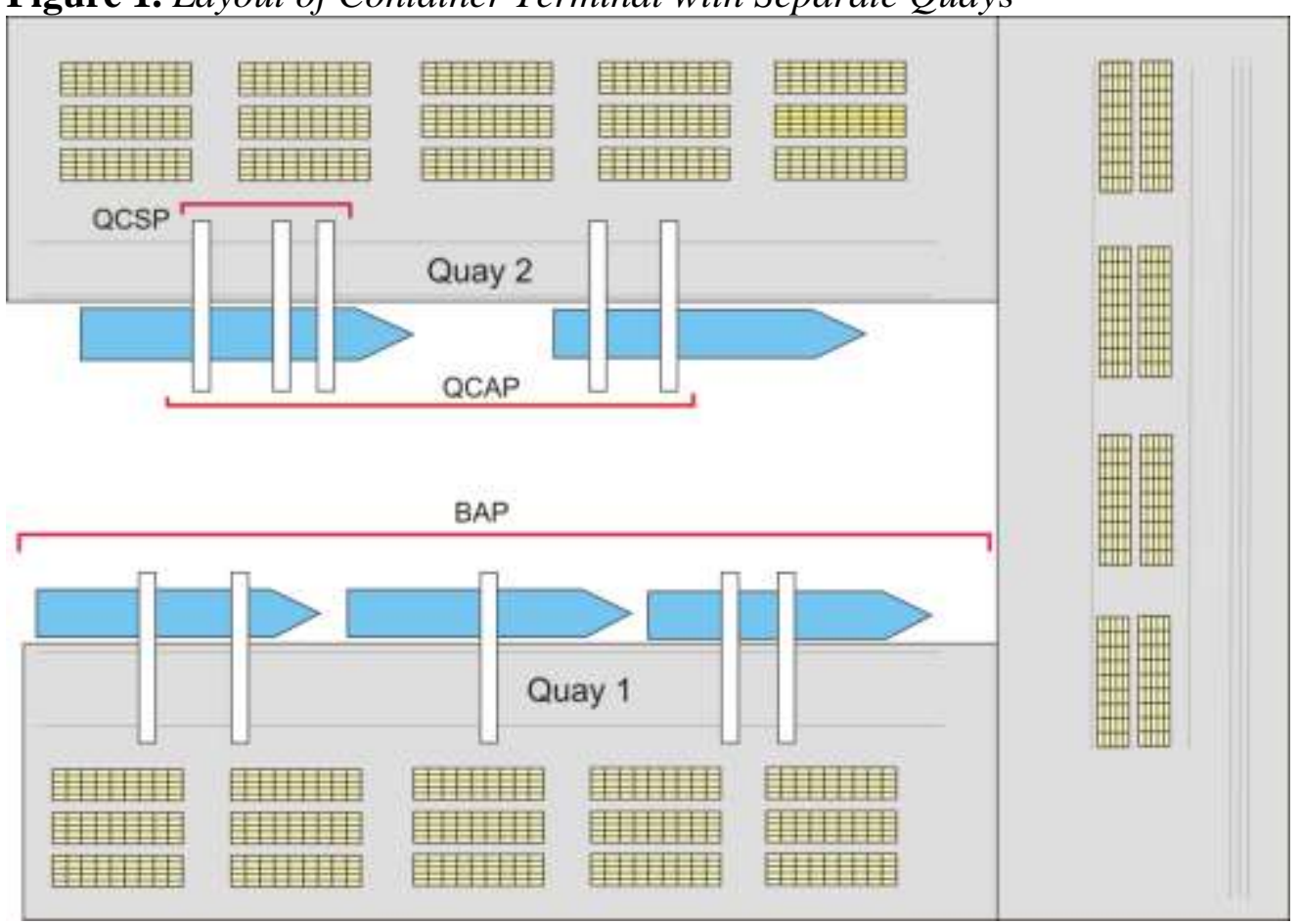

In relation to the size of the terminal, Beskovnik \& Twrdy (2009) suggested four groups of container terminals: small terminals to 500,000 TEUs, a medium-sized terminal from 500 thousand to one million TEUs, large terminals from 1 to 3,000,000 TEUs and largest more than 3 million TEUs. When evaluating the productivity of the medium-sized terminals authors stated the average value of 99,762 TEUs per year per quay-crane and 699 TEUs/1m of the quay length. This would correspond to the terminal configuration with a total quay length of approximately 1430 meters with 10 quay-cranes in the seaside operation system.

Therefore, in the focus of this research are container terminals with two separate quays within the same port basin. The main objective is to how to solve the typical logistic problems in seaside operations to minimize the overall service time for vessels in port and to improve utilization of terminal resources.

A port container terminal may be considered a logistic system (Steenken et al., 2004), where several different technological processes occur with its own tasks, resource assignments and scheduling of operation. There are several tactical logistic problems in the container terminal that must be considered (Rashidi \& 
Tsang, 2006; Murty et. al., 2005). Among them Meisel (2009) grouped three logistic problems targeting seaside operations: Berth Allocation Problem (BAP), Quay Crane Assignment Problem (QCAP) and Quay Crane Scheduling Problem (QCSP).

The Berth Allocation Problem (BAP) is one of the well-known tactical logistic problems in transport process optimization on the container terminals. The problem is to find the optimal assignment of berths to the vessels and to adjust vessel arrivals to the preselected time windows. The objective is to maximize berth capacity and minimize the waiting time for the vessels in the port. The first authors who published papers related to discrete berth problem were Imai et al. (2001) and Nishimura et al. (2001). Lim (1998) carried out research with continuous berth layout.

Another interesting issue is to put various attributes into the objective function according to the terminal business strategy, technical or organizational limitations. Thereby, Kim \& Moon (2003) included a position preference attribute into the model. On the other hand, Guan and Cheung (2004) included a priority coefficient into the model, which varies depending on the vessel characteristics or shipping company. Considering public ports, Imai et al. $(2005 ; 2007)$ presented the priority model based on technical attributes of the terminal subsystems rather than based on particular shipping company. Grubisic (2015) propose modification of the BAP considering draft restrictions.

Quay Crane Assignment is another optimization problem with objective to allocate available cranes to vessels according to the handling demand of the particular vessel. QCAP is closely related to BAP, since the position and time of berthing are input parameters for the crane-to-vessel assignment. This is more elaborated by Zhou \& Kang (2008), and Meisel (2009).

However, the problem with separated or independent quays is not adequately represented in the literature. Only the work of Hendriks et al. (2011) is found corresponding at some extend to this problem. The objective was more balanced quay-crane workload and minimization of the container movement among the terminals. However, authors did not deeply consider the relation between the crane allocation and cargo distribution on board, but approximate the capacity availability.

In the following chapters, we will present an overall approach of the optimization process for berth and quay crane assignment taking into consideration terminal quays layout, cargo distribution on board and variation in vessel handling demand. The goal is to improve the reliability and planning of the operations.

\section{Methodology}

Optimization of the seaside system of the port container terminal combines three separate optimization problems in theory known as the berth allocation problem, the quay-crane assignment problem and the quay-crane scheduling problem. 
The berth allocation problem determines the position and time of berthing the vessel. The main challenge for BAP solving is how to determine the required processing time of the vessel in order to setup an arrival/departure plan for the planning horizon. This processing time includes preparation for handling operations, time for loading/unloading operations and preparation time for departure. Time for loading/unloading operations is primarily important factor and it depends on two key features:

- availability of quay cranes (number of QCs to be assigned)

- transport demand (number of containers) and distribution of container across the ship holds/bays.

The main weakness of many BAP models is the prediction of processing time and implication of that assumption on problem solution. This assumption is closely connected to availability of resources. The schedule of vessels' arrivals is dynamic with variations in regular service operations and it is not always possible to guarantee the availability of the crane at the assigned time-window. If delay occurs for one vessel, most probably it will have impact on overall handling process on the terminal, including crane time-window assignment for other vessels. In that case, the result of the optimization function is not applicable and it is necessary to make reengineering of the process.

Considering this problem, Meisel (2009) proposed an integrated model that solves the BAP and QCAP problem at once. Handling processing time is variable, which value depends on number of assigned cranes and depends on availability of cranes. Moreover, it assumes that the crane capacity is fully exploited. Again, this is not enough because the productivity of the handling and a crane itself depends on the cargo distribution on board. It is not possible to utilize crane capacity and evenly arrange the workload of cranes for particular ship if we use only one model approach. Therefore, Meisel included the quaycrane scheduling problem in the optimization process to be performed before the berth/crane assignment based on cargo distribution data and cargo plans.

Regarding the properties of the individual models for BAP, QCAP and QCSP we have developed the methodology by which it would be possible to provide a complete solution as a result of the optimization process, not a single model. Because of the interdependence of the result of the decision variables and input parameters, individual models that have, a high correlation with each other should be considered together. This optimization process is based on the integration and interdependence of individual optimization problems (Figure 2 ). The feature of this optimization process is operational functionality of targeted medium-size container terminals with separated quays.

The process consists of three main stages: initiation, allocation and adjustment. In the initiation stage, handling sequences and crane arrangement are modeled based on vessels and cargo data. The result of the initiation stage, are three options of QC arrangement for each vessel, which are chosen as reliable and possible after solving the quay-crane scheduling problem. The results strictly follow containers distribution on board each vessel. 
The core of the second stage is an optimal solution finding for ship to berth and crane to ship allocation. Therefore, the models for solving BAP and QCAP for separate quays are generated where the relocation of resources is performed by the verification of three possible options resulting from the initiation stage. The objective of this stage is to find the overall optimal solution for the minimization of the vessels service time in port and maximum utilization of cranes service times.

The last stage of the process is the adjustment and alignment of the vessel arrival schedule where time-window reservation principle may be implemented according to mutual agreement with shipping companies and a terminal operator (Zuskin et al., 2015). In addition, in this stage it is possible to rearrange the berthing plan in case the two adjacent planning horizons are overlapping.

The final tactical-operational plan integrates berth allocation to vessels, timetable for berthing, assignment of fixed number of quay cranes per each vessel, quay-crane timetable of workload and tasks sequencing.

Figure 2. Optimization Process for Seaside Operations on CT

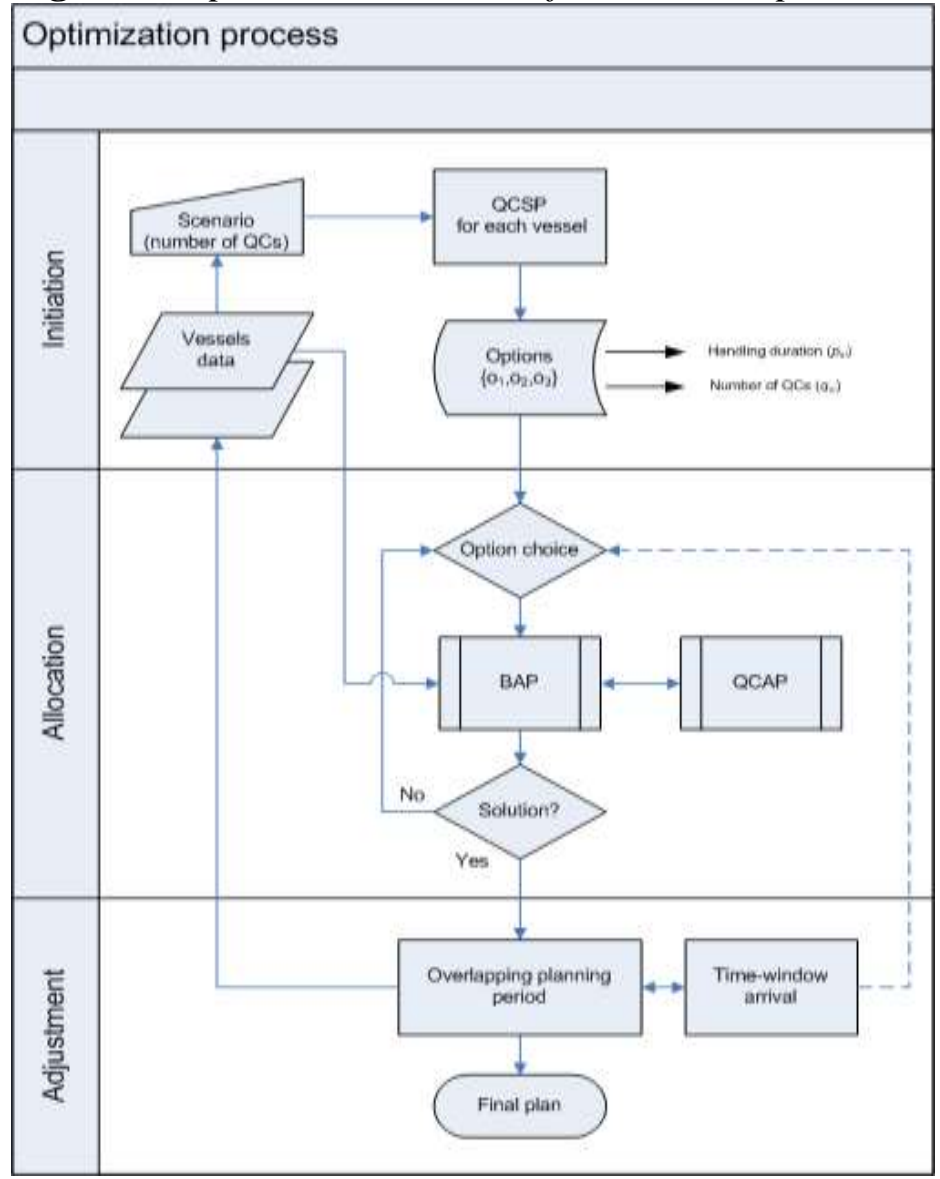




\section{Model Formulation}

\section{Initiation - Handling Scenario Determination}

The optimization process begins with the initiation stage. Based on the vessel arrival data and cargo distribution on board, three-operation scenarios are developed according to the results of the quay-crane scheduling problem solutions. For the quay-crane scheduling modeling two different models are used, developed in the previous work (see Grubisic \& Dundovic, 2014). Each option is defined by variable $O(q, p)$ where $q$ and $p$ are parameters describe number of reserved cranes and service time necessary for loading/unloading operations. Input and output variables for initiation are shown on Figure 3.

Figure 3. Operational Scenario as Results of QCSP Solution

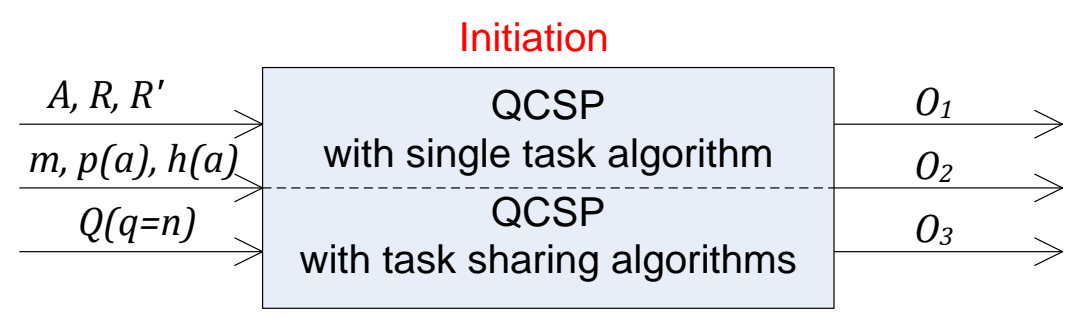

For each QCSP problem solution, it is necessary to define $q$ beforehand. The minimum number of quay cranes $q_{i}^{\min }$ available for each vessel is usually subject to a contract between terminal operator and shipping company while the maximum number of quay cranes $q_{i}^{\max }$ depends on cargo distribution. In cases where $q=1$ the problem is limited to solving a task sequence only. In other cases, QCSP solution determines task-to-crane assignment and total makespan $p_{i}$ required for completion of handling operations. For each vessel $i$ operational scenario is defined by the expression:

$$
\mathbf{O}=\left[\begin{array}{ll}
q_{1} & p_{1} \\
q_{2} & p_{2} \\
q_{3} & p_{3}
\end{array}\right]
$$

According to the three different processing times $p$ are obtained depending on the number of quay-crane assignment $q$. Which option would be the best option depends on results of forthcoming optimization process in allocation stage. In initiation stage, it is important to carry out the QCSP solving for each $q$ in interval $\left[q^{\min }, q^{\max }\right]$.

The key feature of the proposed model are the output values that have three possible options for crane assignment determining the total duration of the handling process. It should be pointed out that these three options have balanced the operation time and at most even distribution of workload. This is achieved by implementation of task sharing algorithm for QCSP solution (Grubisic \& Dundovic, 2014). This specific feature ensures a high utilization 
rate of quay cranes and allows the simplification of the optimization problem as we can consider fixed number of quay cranes during entirely handling process. It is only necessary to choose between the three options. This simplifies the overall optimization process and avoids the uncertainty that occurs when crane to ship allocation is based on time-windows assignment. On the other hand, from the shipping company point of view, the approach when a number of cranes is fixed during the entirely loading/unloading process is much more acceptable and port services much more reliable.

\section{Allocation - Quay, Berth and Crane Assignment}

In this stage, we are solving integrated BAP and QCAP model adapted for terminals with separate quays layout configuration. Input parameters for the model are the length and arrival time of the vessels together with values of output variables from the initiation stage. In addition, for quay choice we define set of quays $W=\left\{W_{1}, W_{2}, \ldots, W_{K}\right\}$ with index $k \leq K$ each with total quay length $Q L_{k}$ and total number of cranes $Q C_{k}$.

In practice, shipping companies have preferences over the particular quay choice. Before vessel arrival, containers are prepared in blocks near the berth positions located at the preferred quay. If there is a change in quay assignment, it will more likely result in more resources engagement for reposition of the containers and consequently generate additional costs.

In order to satisfy the requirements of preference-based selection of quay, it is necessary to penalize berth at the quay, which is less favorable for the vessel or would lead to an increase of the cost for reposition of the containers on the terminal. If the cost of reposition is marked with $c p$ and the unit cost per time unit of the ship waiting for the free berth is marked with $c w$, then the ratio $c p / c w$ is "quay weight factor" marked with $\omega_{i k}$. Its value is determined depending on the vessel preference, based on costs $c p$ and $c w$, according to the following expression:

$$
\omega_{i k}=\frac{c p_{i k}}{c w_{i}} \quad z a \forall i \in V, \forall k \in W
$$

Quay weight factor is relative dimension indicates the ratio of cost for the ship repositioning in relation to the cost of the ship waiting for the free berth. The integrated model for BAP and QCAP optimization for separate quays is shown below.

We define the following sets:

$V=\left\{V_{1}, V_{2}, \ldots, V_{N}\right\}$ is set of vessels with index $i \in \square: i \leq N$, where $N$ is total number of vessels in the system. $T=\left\{T_{1}, T_{2}, \ldots, T_{H}\right\}$ is set of time windows with index $t \in \square: t \leq H$, where $H$ is planning horizon. $W=\left\{W_{1}, W_{2}, \ldots, W_{K}\right\}$ 
is set of quays with index where $K$ is number of quays. $O=\left\{O_{1}, O_{2}, O_{3}\right\}$ is set of handling scenario (options) with index $v$.

Following notation is applied:

$w_{i}$ - waiting time of vessel $i$ for free berth

$p_{i v}$ - duration of handling process for vessel $i$ in scenario $v$

$\omega_{i k}$ - quay weight factor for vessel $i$ to be docked at quay $k$

Binary variable used in the model are:

$o_{i v}= \begin{cases}1, & \text { if scenario } v \text { is chosen for vessel } i \\ 0, & \text { otherwise }\end{cases}$

$x_{i k}= \begin{cases}1, & \text { if vessel } i \text { is assigned to quay } k \\ 0, & \text { otherwise }\end{cases}$

The objective function minimizes port service time for the vessels to be processed at the container terminal with separate quays and read:

$$
\operatorname{MinZ}=\sum_{i \in V} w_{i}+\sum_{i \in V} \sum_{v \in O} p_{i v} o_{i v}+\sum_{i \in V} \sum_{k \in W} x_{i k} \omega_{i k}
$$

The allocation of resources is achieved by selecting one among the three predefined scenario with objective to get minimum value of the function. Shipto-berth assignment is optimized on the same manner as in conventional BAP problem solutions. The difference is in vessel-processing time that is not fixed but depends on the chosen handling scenario. The model is designed such a way that the best option for the vessel is always taken at first. That is the one with the highest number of quay cranes and the shortest processing time. In this way, the QCAP solution comes down to the selection of the scenario rather than individually assignment of the cranes. As the basis for QCAP solution is outcome of QCSP solution, together with the implementation of the split task algorithm, the uncertainty resulting from the crane demand approximation is suppressed. The best scenario selection for the particular vessel typically leads to the lack of a real solution if resources (quay cranes) are limited. In that case, the model selects another operating scenario for each vessel to get integrated and optimal solution for the planning horizon.

\section{Adjustment}

Two situations during the planning horizon may occur, both as result of variation in vessel schedule. First, it may happen that the vessel arrival is scheduled at the end of the planning period. If this happens and the vessel 
handling process continues after the end of the planning horizon, adjustments should be made in order to avoid the double allocation of berths to vessels that are scheduling at the beginning of the next planning period. The second situation is when it is possible to adjust the schedule of the vessel according to a mutual agreement between the shipping company and terminal operator. The idea behind is that the optimization result may be improved by speed-up or slow-down the vessel voyage in order to fit her ETA into the reserved timewindows. If applicable, adjustment means that optimization process need to restart with the adjusted input values of vessels parameters.

To perform the adjustment based on mutual agreement between a terminal operator and a shipping company it is necessary to modify the objective function so that it reads:

$$
\operatorname{Min} Z=\sum_{i \in V}\left[\left(w_{i} \omega_{i}^{w a i t}+e_{i} \omega_{i}^{a r r}\right)+\sum_{v \in O} p_{i v} o_{i v}+\sum_{k \in W} x_{i k} \omega_{i k}\right]
$$

where new notations are:

$e_{i}$ - time saving or difference between estimated and earlier time of arrival

$\omega_{i}^{\text {wait }}$ - weighted coefficient of waiting for free berth

$\omega_{i}^{a r r}$ - weighted coefficient of the vessel earlier arrival at the port

\section{Application Example}

In the following examples we demonstrate how to solve BAP and QCAP problems on the container terminal with two separate quays (or piers) when 7 ships are scheduled for arrival during the planning horizon. Planning horizon may be arbitrary selected, usually on the weekly basis.

Each vessel competes equally for available berths but with preference of quay 1 over the quay $2\left(\omega_{i 1}=1, \omega_{i 2}=5\right)$. Both weighted coefficients, for waiting and for earlier arrival, are the same for all vessels in this simple example $\left(\omega_{i}^{\text {wait }}=1, \omega_{i}^{a r r}=2\right)$. For both quays, the total length is expressed in the number of berth segments and is set to 15 . Let us say each berth segment has a length of 50 meters that corresponds to 750 meters. There are 5 quay cranes available on each quay. Values for $p_{i k}$ and $q_{i k}$ for scenarios $o_{i v}$ are calculated after QCSP solution is found in initiation stage. Processing time for handling operations $p_{i k}$ are expressed in time-window segments. The input parameters are shown in Table 1 and solution based on objective function (4) is shown in Table 2 and Figure 4. 
Table 1. Example 1: Input Data

\begin{tabular}{|c|c|c|c|c|c|c|c|c|c|}
\hline$i$ & V & $l_{i}$ & $a_{i}$ & $a_{i}{ }^{\prime}$ & $\omega_{i}^{\text {wait }}$ & $\omega_{i}^{\text {arr }}$ & $p_{i 1} / p_{i 2} / p_{i 3}$ & $q_{i 1} / q_{i 2} / q_{i 3}$ & $\omega_{i 1} / \omega_{i 2}$ \\
\hline 1 & $\mathrm{~A}$ & 6 & 1 & 1 & 1 & 2 & $5 / 6 / 10$ & $4 / 3 / 2$ & $1 / 5$ \\
\hline 2 & B & 6 & 3 & 3 & 1 & 2 & $4 / 5 / 8$ & $3 / 2 / 1$ & $1 / 5$ \\
\hline 3 & $\mathrm{C}$ & 4 & 9 & 8 & 1 & 2 & $9 / 13 / 25$ & $3 / 2 / 1$ & $1 / 5$ \\
\hline 4 & $\mathrm{D}$ & 4 & 6 & 6 & 1 & 2 & $6 / 10 / 20$ & $3 / 2 / 1$ & $1 / 5$ \\
\hline 5 & $\mathrm{E}$ & 7 & 12 & 12 & 1 & 2 & $6 / 7 / 10$ & $5 / 4 / 3$ & $1 / 5$ \\
\hline 6 & $\mathrm{~F}$ & 4 & 15 & 12 & 1 & 2 & $4 / 8 / 1000$ & $2 / 1 / 1000$ & $1 / 5$ \\
\hline 7 & $\mathrm{G}$ & 5 & 10 & 8 & 1 & 2 & $5 / 7 / 11$ & $4 / 3 / 2$ & $1 / 5$ \\
\hline$k$ & \multicolumn{2}{|c|}{$W L_{k}$} & \multicolumn{2}{|c|}{$Q C_{k}$} & & & & & \\
\hline 1 & \multicolumn{2}{|c|}{15} & \multicolumn{2}{|c|}{5} & & & & & \\
\hline 2 & \multicolumn{2}{|c|}{15} & \multicolumn{2}{|c|}{5} & & & & & \\
\hline
\end{tabular}

The following notations are used in the Table 1:

$V$ - vessel name

$l_{i}$ - length of the vessel $i$ (expressed in quay segments)

$a_{i}$ - estimated time of vessel arrival (ETA)

$a_{i}{ }^{\prime}$ - the earliest possible estimated time of the vessel arrival

$\omega_{i}^{\text {wait }}, \omega_{i}^{\text {arr }}$ - weighted coefficients related to cost

$p_{i 1} / p_{i 2} / p_{i 3}$ - processing time for handling operations for three scenarios

$q_{i 1} / q_{i 2} / q_{i 3}$ - number of QCs assigned to vessel $i$ for three scenarios

$\omega_{i 1} / \omega_{i 2}-$ quay weight factor for vessel $i$ for a corresponding quay

$W L_{k}$ - total quay length (in the number of berth segments)

$Q C_{k}$ - total number of quay cranes located at the quay $k$

Table 2. Example 1: Results of Integrated Optimization Model

\begin{tabular}{|c|c|c|c|c|c|c|c|c|}
\hline$i$ & $V$ & $b_{i}$ & $s_{i}$ & $d_{i}$ & $w_{i}$ & $e_{i}$ & $x_{i 1} / x_{i 2}$ & $o_{i 1} / o_{i 2} / o_{i 3}$ \\
\hline 1 & $\mathrm{~A}$ & 1 & 1 & 7 & - & - & $1 / 0$ & $0 / 1 / 0$ \\
\hline 2 & $\mathrm{~B}$ & 10 & 3 & 8 & - & - & $1 / 0$ & $0 / 1 / 0$ \\
\hline 3 & $\mathrm{C}$ & 5 & 15 & 24 & 6 & - & $1 / 0$ & $1 / 0 / 0$ \\
\hline 4 & $\mathrm{D}$ & 1 & 6 & 12 & - & - & $0 / 1$ & $1 / 0 / 0$ \\
\hline 5 & $\mathrm{E}$ & 9 & 12 & 18 & - & - & $0 / 1$ & $1 / 0 / 0$ \\
\hline 6 & $\mathrm{~F}$ & 1 & 15 & 19 & - & - & $1 / 0$ & $1 / 0 / 0$ \\
\hline 7 & $\mathrm{G}$ & 11 & 10 & 15 & - & - & $1 / 0$ & $1 / 0 / 0$ \\
\hline \multicolumn{7}{l}{ Min Z 62}
\end{tabular}


Following notations are used in the Table 2:

$b_{i}$ - berth position (number of first segment assigned to vessel $i$ )

$s_{i}$ - berthing time (first time-window assigned to vessel $i$ )

$d_{i}$ - departure time of vessel $i$

$w_{i}$ - waiting time of vessel $i$

$e_{i}$ - time saving for earlier arrival of vessel $i$

Figure 4. Graphical Representation of Solution for Example 1

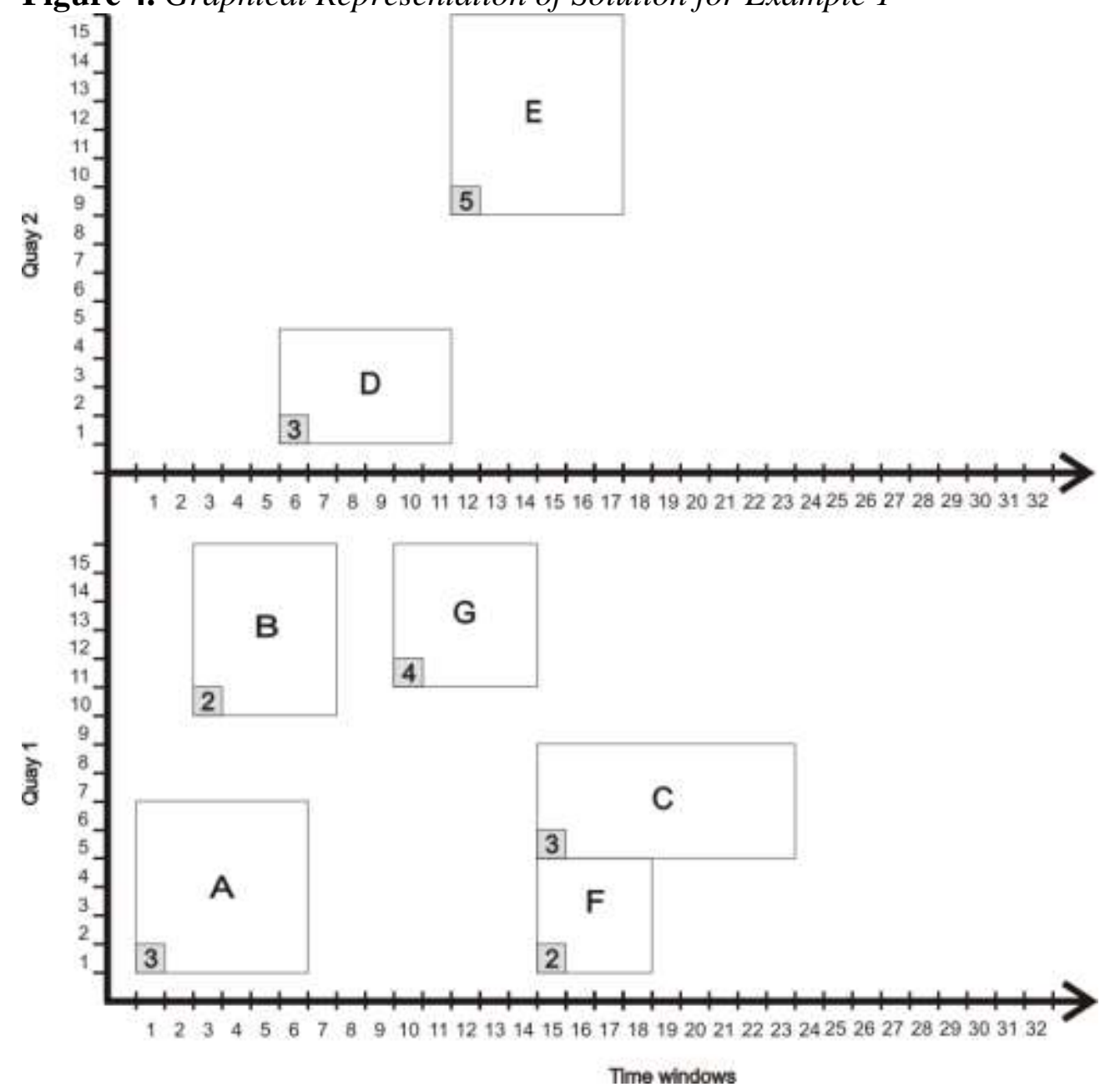

The graphical solution of the integrated optimization problem is presented in Figure 4. Vessels are represented by the rectangle with time-windows on xaxis and berth segments on y-axis. The number of assigned cranes is shown in down-left corner of the rectangle.

The optimal solution of the objective function (4) is 62. Vessels D and E are berthed alongside the quay number 2 where maximum number of QCs is assigned to both of them according to the best of three possible handling scenarios. Vessel $\mathrm{C}$ is only one who has to wait for a free berth at the quay 1 , but after 
berthing maximum number of QCs will be assigned in order to speed-up the processing time for handling operations. Earlier berthing time for vessel $\mathrm{C}$ would not result in optimal solution because it would be not being possible to allocate 3 but only 1 or 2 cranes, that will prolong the processing time from 9 to 13 or even 25 time windows, according to QCSP solution. Figure 4 shows that number of assigned cranes corresponds to the selected scenarios for each vessel and does not exceed the maximum number of QCs at the quay. The number of cranes assigned to the vessels is constant during the overall handling process. This has avoided the impact of the handling operations taking place on other vessels nearby on availability of QCs that may occur in case when the number of QCs assigned to the vessel is variable.

Let us consider the second example that explains the third stage of the optimization process - the adjustment stage. In addition to the previous example, we put three more vessels in the system, namely $\mathrm{P}, \mathrm{R}$ and $\mathrm{S}$ which arrivals are scheduled in next planning horizon, which is arbitrary selected at time window 16 (see Figure 5). The input data for these vessels are shown in Table 3.

Table 3. Example 2: Input Data

\begin{tabular}{|l|l|l|l|l|l|l|l|l|l|}
\hline$i$ & $V$ & $l_{i}$ & $a_{i}$ & $a_{i}{ }^{\prime}$ & $\omega_{i}{ }^{\text {wait }}$ & $\omega_{i}{ }^{\text {arr }}$ & $p_{i 1} / p_{i 2} / p_{i 3}$ & $q_{i 1} / q_{i 2} / q_{i 3}$ & $\omega_{i 1} / \omega_{i 2}$ \\
\hline 1 & $\mathrm{P}$ & 7 & 1 & 1 & 1 & 2 & $5 / 6 / 10$ & $3 / 2 / 1$ & $1 / 5$ \\
\hline 2 & $\mathrm{R}$ & 6 & 3 & 3 & 1 & 2 & $4 / 5 / 8$ & $3 / 2 / 1$ & $1 / 5$ \\
\hline 3 & $\mathrm{C}$ & 4 & $\mathbf{1}$ & $\mathbf{1}$ & 1 & 2 & $\mathbf{7 / 1 3 / 2 5}$ & $3 / 2 / 1$ & $1 / 5$ \\
\hline 4 & $\mathrm{~S}$ & 5 & 5 & 5 & 1 & 2 & $6 / 8 / 10$ & $3 / 2 / 1$ & $1 / 5$ \\
\hline 5 & $\mathrm{E}$ & 7 & $\mathbf{1}$ & $\mathbf{1}$ & 1 & 2 & $\mathbf{1} / 7 / 10$ & $5 / 4 / 3$ & $1 / 5$ \\
\hline 6 & $\mathrm{~F}$ & 4 & $\mathbf{1}$ & $\mathbf{1}$ & 1 & 2 & $\mathbf{2} / 8 / 1000$ & $2 / 1 / 1000$ & $1 / 5$ \\
\hline
\end{tabular}

Considering the optimal solution obtained from previous stages, we can notice that vessels $\mathrm{C}, \mathrm{E}$ and $\mathrm{F}$ exceed the actual planning horizon and should be included in the next one. However, they are already berthed and the handling operations continue at the beginning of the second planning horizon. Therefore, we must take into account the time remaining for completion of handling operations and fix their positions in time-space diagram. This has been done by setting fixed values for variables $b_{i}, a_{i}$ and $s_{i}$ such that $b_{3}=5, b_{5}=9, b_{6}=1$, $a_{3}=a_{5}=a_{6}=1$, and $s_{3}=s_{5}=s_{6}=1$. Binary decision variables must be fixed such that $x_{3,1}=1, x_{5,2}=1$, and $x_{6,1}=1$. Values for $p_{3,1}, p_{5,1}, p_{6,1}$ should be adjusted accordingly. 
Table 4. Example 2: Output Data

\begin{tabular}{|c|c|c|c|c|c|c|c|c|}
\hline$i$ & $V$ & $b_{i}$ & $s_{i}$ & $d_{i}$ & $w_{i}$ & $e_{i}$ & $x_{i 1} / x_{i 2}$ & $o_{i 1} / o_{i 2} / o_{i 3}$ \\
\hline 1 & $\mathrm{P}$ & 1 & 2 & 7 & 1 & - & $0 / 1$ & $1 / 0 / 0$ \\
\hline 2 & $\mathrm{R}$ & 9 & 3 & 8 & - & - & $1 / 0$ & $0 / 1 / 0$ \\
\hline 3 & $\mathrm{C}$ & $\mathbf{5}$ & $\mathbf{1}$ & 8 & - & - & $\mathbf{1} / 0$ & $\mathbf{1} / 0 / 0$ \\
\hline 4 & $\mathrm{~S}$ & 9 & 8 & 14 & 3 & - & $1 / 0$ & $1 / 0 / 0$ \\
\hline 5 & $\mathrm{E}$ & $\mathbf{9}$ & $\mathbf{1}$ & 2 & - & - & $0 / \mathbf{1}$ & $\mathbf{1} / 0 / 0$ \\
\hline 6 & $\mathrm{~F}$ & $\mathbf{1}$ & $\mathbf{1}$ & 3 & - & - & $\mathbf{1} / 0$ & $\mathbf{1} / 0 / 0$ \\
\hline
\end{tabular}

Figure 5. Graphical Representation of Solution for Example 2

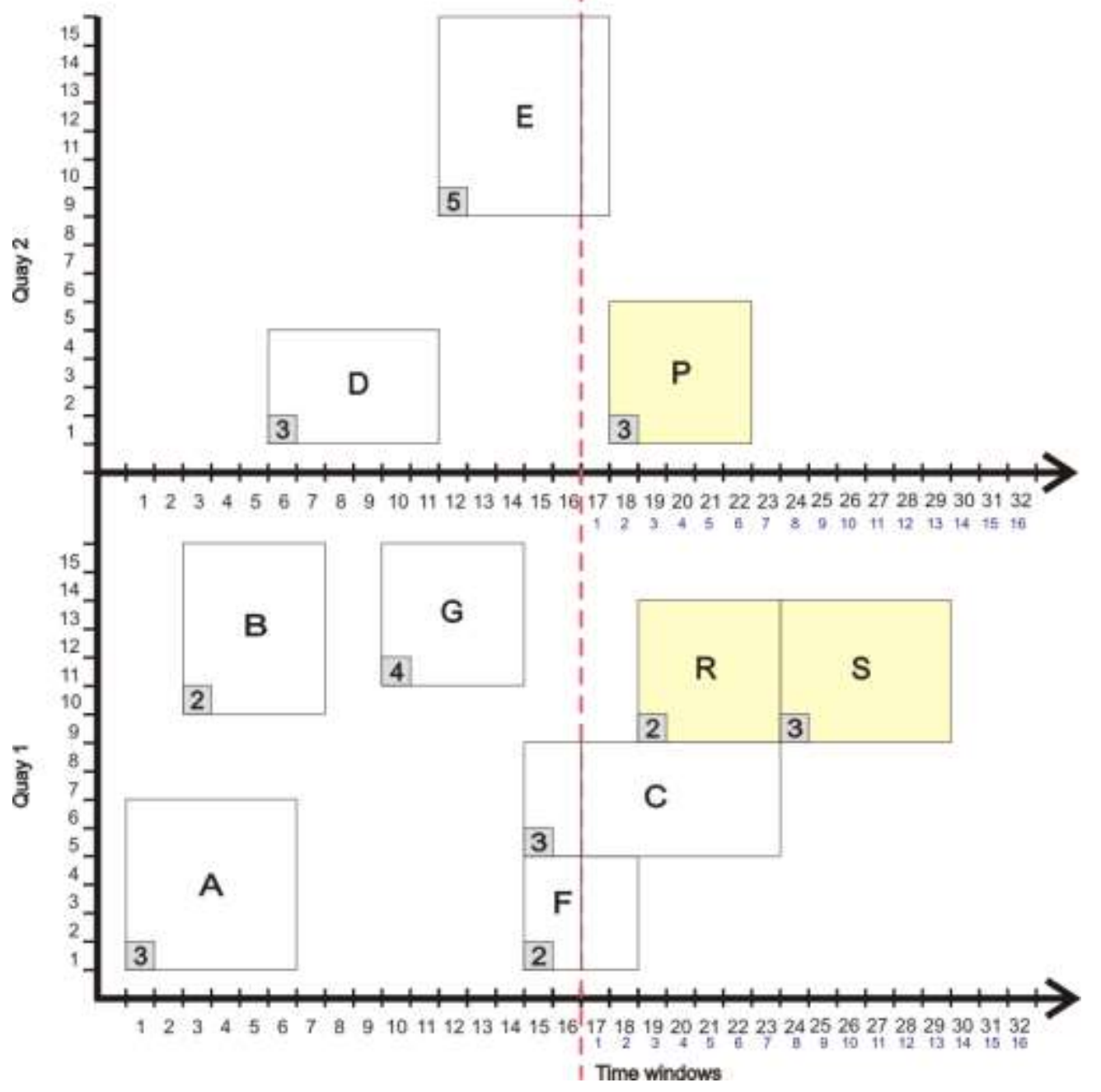


It can be concluded that the results fully correspond to the given condition in terms of allocation of the vessels from previous planning horizons and berth and time-window assignment of new vessels entering the system in new planning period (Table 4). For example, vessel $\mathrm{P}$ has to wait completion of operations on vessel $\mathrm{E}$ because there is no QC available for operations. None of the vessels $\mathrm{P}, \mathrm{R}$ or $\mathrm{S}$ is allocated to the berthing positions occupied by the vessels $\mathrm{C}, \mathrm{E}$ and $\mathrm{F}$ or violates the rule of maximum number of $\mathrm{QCs}$ at the quays (Figure 5).

Vessel $\mathrm{S}$ in this example must wait three time-windows because it is not possible to find better solution. If we place $S$ on the quay number 2 , above the $\mathrm{P}$ immediately after the arrival, due to quay weight factor the total value of objective function will be higher. On the other side, looking at the vessel R, only 2 QCs are possible to be assigned choosing the scenario 2 for the handling operations.

\section{Conclusions}

Typical logistic problems on container terminals known as BAP, QCAP and QCSP can be integrated to solve different issues and requirements of optimization. The model proposed in this research target different terminal layouts trying to solve complex correlation between those three problems. From the present experience, that integration is possible with some assumptions regarding the quay crane capacity planning. One may notice that fixed number of cranes per vessel during the entirely handling process is weak point of the model. However that is a question of terminal policy where both sides, terminal operator and shipping companies, may benefit from better prediction of the service time and the service reliability. The key for the model success is high utilization rate and good balance between workloads of quay cranes assigned to each vessel that need to be achieved by implementation of split-task algorithm developed in previously research. Further action should be directed to the model testing for different intervals of vessel's arrival and for different container distribution on board the vessel.

\section{References}

Beskovnik, B. \& Twrdy, E. (2009) Productivity simulation model for optimization of Maritime Container Terminals. Transport Problems. 4 (3), pp. 113-122.

Grubisic, N., Dundovic, C. (2014) A Solution for Container Terminal QC Scheduling Considering Grouped Tasks and Operative Zone Limits. ICIL 2014 Conference Proceedings/Lesar, Mario (ed.). International Conference on Industrial Logistics, Bol, Croatia.

Grubisic, N. (2015) A Contribution to Berth Allocation Problem Solution with Draft Restrictions. Pomorski zbornik. 49-50 (1), pp.127-142.

Guan, Y. \& Cheung, R.K. (2004) The berth allocation problem: models and solution methods. OR Spectrum. 26 (1), pp. 75-92. 
Hendriks, M.P.M., Armbruster, D., Laumanns, M., Lefeber, E. \& Udding, J.T. (2011) Strategic allocation of cyclically calling vessels for multi-terminal container operators. Flexible Services and Manufacturing Journal.

Imai, A., Nishimura, E. \& Papadimitriou, S. (2001) The dynamic berth allocation problem for a container port. Transportation Research Part B: Methodological. 35 (4), pp. 401-417.

Imai, A., Sun, X., Nishimura, E. \& Papadimitriou, S. (2005) Berth allocation in a container port: using a continuous location space approach. Transportation Research Part B: Methodological. 39 (3), pp. 199-221.

Imai, A., Nishimura, E., Hattori, M. \& Papadimitriou, S. (2007) Berth allocation at indented berths for mega-containerships. European Journal of Operational Research. 179 (2), pp. 579-593.

Kim, K.H. \& Moon, K.C. (2003) Berth scheduling by simulated annealing. Transportation Research Part B: Methodological. 37 (6), pp. 541-560.

Lim, A. (1998) The berth planning problem. Operations Research Letters. 22 (2-3), pp. 105-110.

Meisel, F. (2009) Seaside Operations Planning in Container Terminals. Springer.

Murty, K.G., Liu, J., Wan, Y. \& Linn, R. (2005) A decision support system for operations in a container terminal. Decision Support Systems. 39 (3), pp. 309-332.

Nishimura, E., Imai, A. \& Papadimitriou, S. (2001) Berth allocation planning in the public berth system by genetic algorithms. European Journal of Operational Research. 131 (2), pp. 282-292.

Rashidi, H. \& Tsang, E.P.K. (2006) Container Terminals: Scheduling Decisions, their Formulation and Solutions.

Steenken, D., Voss, S. \& Stahlbock, R. (2004) Container terminal operation and operations research - a classification and literature review. OR Spectrum. 26 (1), pp. 3-49.

Zhou, P. \& Kang, H. (2008) Study on Berth and Quay-crane Allocation under Stochastic Environments in Container Terminal. Systems Engineering - Theory \& Practice. 28 (1), pp. 161-169.

Zuskin, S., Grubisic, N., Sumner, M. (2015) Shipowner management in accordance with mutual agreement. Pomorstvo: Scientific Journal of Maritime Research. 29 (1), pp. 69-74. 
